\title{
From Generation to Extraction: a Time-Resolved Investigation of Photophysical Processes in Non-Fullerene Organic Solar Cells
}

\author{
Rokas Jasiūnas ${ }^{1}$, Huotian Zhang ${ }^{3}$, Jun Yuan ${ }^{4}$, Deping Qian ${ }^{3}$, Yingping Zou ${ }^{4}$, Andrius Devižis ${ }^{1}$, \\ Juozas Šulskus ${ }^{2}$, Feng Gao ${ }^{3}$ and Vidmantas Gulbinas*1,2 \\ 1 Center for Physical Sciences and Technology, Sauletekio av.3, Vilnius, 10257 (Lithuania) \\ 2 Institute of Chemical Physics, Faculty of Physics, Vilnius University, Sauletekio av. 9-III, 10222 Vilnius \\ (Lithuania) \\ 3 Department of Physics Chemistry and Biology (IFM), Linköping University, Linköping SE-58183, Sweden \\ 4 College of Chemistry and Chemical Engineering, Central South University, Changsha 410083, P.R. China
}

\section{Suplementary Information}

\section{Materials and Devices.}

Material and Device: PBDB-T, PBDB-T-2Cl, ITIC were purchased from Solarmer. PC ${ }_{71} B M$ was purchase from Solennebv. PDCBT-2F was synthesized at the Institute of Chemistry, Chinese Academy of Sciences. $\mathrm{Y} 1$ was synthesized at the Central South University. The zinc oxide ( $\mathrm{ZnO}$ ) solution Avantama N10 was purchased from Avantama AG. Other materials were all synthesized as reported previously. The device structure for all blends is ITO/ $\mathrm{ZnO} /$ active layer/ $/ \mathrm{MoO}_{3} / \mathrm{Al}$, with an effective area of $4.7 \mathrm{~mm}^{2}$. The ITO glass substrates were cleaned with detergent, distilled water, acetone and isopropanol for 15 minutes in sequence. Then the substrates were dried with nitrogen gas flow and treated with UV-ozone for 15 minutes. ZnO solution was used as purchased and spin coated on the ITO glass at $3000 \mathrm{rpm}$, followed by 30-mintute annealing at $120^{\circ} \mathrm{C}$ in ambient air. The substrates with $\mathrm{ZnO}$ were treated with $254 \mathrm{~nm}$ UV light for 15 minutes in glovebox filled with nitrogen atmosphere before use. PBDB-T:PC 71 BM were dissolved in $\mathrm{CB}$ with 1,8-diiodooctane(DIO) (3\%, volume ratio, added 30 minutes before use) as a 1:1.5 mixture of $20 \mathrm{mg} / \mathrm{ml}$ in total and stirred for 8 hours $\left(60^{\circ} \mathrm{C}\right)$. PBDB-T:Y1 (D:A = 1:1, weight ratio, $16 \mathrm{mg} / \mathrm{ml}$ in total) were dissolved in chlorobenzene (CB) and 1-chloronaphthalene (CN) (0.8\%, volume ratio) and stirred for 8 hours $\left(80^{\circ} \mathrm{C}\right)$. PBDB-T-2Cl:ITIC were dissolved in CB as a $1: 1$ mixture of $20 \mathrm{mg} / \mathrm{ml}$ in total and stirred for 8 hours. PDCBT-2F:ITIC (D:A $=1.5: 1$, weight ratio, $16 \mathrm{mg} / \mathrm{ml}$ in total) were dissolved in chloroform (CF) with CN $(0.5 \%$, volume ratio) and stirred for 2 hours. The active layer of PBDB-T:PCBM, PBDB-T:Y1, PBDB-T-2CI:ITIC and PDCBT-2F:ITIC were spin coated in the glovebox at $3000 \mathrm{rpm}, 2000 \mathrm{rpm}$, $3000 \mathrm{rpm}$ and $3000 \mathrm{rpm}$ respectively. After all the substrates with active layers were annealed at $100{ }^{\circ} \mathrm{C}$ for $10 \mathrm{~min}$, a $5 \mathrm{~nm} \mathrm{MoO3}$ layer and a $100 \mathrm{~nm} \mathrm{Ag}$ layer were subsequently thermally evaporated at $10^{-6}$ mbar through a shadow mask to define the active area of the devices. J-V curves (measured in the forward direction, that is, from negative to positive bias, with a scan step of $0.04 \mathrm{~V}$ ) were collected using a Keithley 2400 Source Meter under AM1.5 illumination provided by a solar simulator (LSH-7320 ABA 
LED solar simulator) with an intensity equivalent to $1,000 \mathrm{~W} \mathrm{m-2}$ after spectral mismatch correction. The light intensity for the J-V measurements was calibrated with a reference Si cell (VLSI standards SN 10510-0524 certified by National Renewable Energy Laboratory). All the photophysical measurements on blends were carried out over the same devices for J-V curve measurement. The transient absorption for pristine PBDB-T and Y1 was conducted on pure films on glass The pristine-material films were spin coated at $3000 \mathrm{rpm}$ with $12 \mathrm{mg} / \mathrm{ml} \mathrm{CF}$ solution followed $100^{\circ} \mathrm{C}$ annealing for 10 minutes in glovebox.

\section{Experimental section.}

2.1 Transient Photocurrent and TDFC Measurements. Transient photocurrent and time delayed collection field investigations were performed with an Agilent Technologies DS05054A oscilloscope using $50 \Omega$ input resistor and a Tektronix AFG 3101 function generator. Samples were excited by radiation of the optical parametric amplifier Topas-C (Light Conversion Ltd.) pumped by femtosecond Ti:sapphire laser Integra-C from Quantronix Inc. generating $130 \mathrm{fs}$ duration pulses at $430 \mathrm{~Hz}$ repetition rate. Collinear optical parametric amplifier TOPAS-C was used for the generation of the excitation pulse emitting at $515 \mathrm{~nm}$.

2.2 Transient Absorption Spectroscopy. The transient absorption setup was based on the amplified femtosecond laser Pharos 10 - 600 - PP laser (Light Conversion Ltd.), operating at fundamental wavelength of $1032 \mathrm{~nm}$, repetition rate of $200 \mathrm{kHz}$, and pulse width of $<250 \mathrm{fs}$. The measurements were performed at repetition rate of $200 / 42=4.762 \mathrm{kHz}$ frequency achieved by using the pulse picker. The collinear optical parametric amplifier Orpheus PO15F2L (Light Conversion Ltd.) was used to obtain 515 $\mathrm{nm}$ or $730 \mathrm{~nm}$ wavelength pulses for sample excitation. Excitation was modulated at $4.762 / 6=0.794 \mathrm{kHz}$ frequency by mechanical chopper synchronized to the output of the pulse picker. As a probe, pulses were used, spectrally broadened by means of continuum generation in the sapphire crystal. The detection equipment consisted of Andor - Shamrock SR - 500i - B1 - R spectrometer (Andor Technology, 150 lines mm-1 diffraction grating) equipped with Andor - Newton (Andor Technology) DU970 CCD camera (1600 $\times 200$ pixels). The reading of the camera was synchronized with the chopper.

2.3 Time resolved optical electric field probing. The experimental setup used for the OEFP measurements was implemented on a conventional, above described, femtosecond absorption pumpprobe spectrometer. More details can be found in [1], [2].

2.4 UV-Vis absorption. UV-Vis absorption spectra were recorded with a Jasco V-670 spectrophotometer in the range of $300-900 \mathrm{~nm}$. 
3. Supporting characterization data

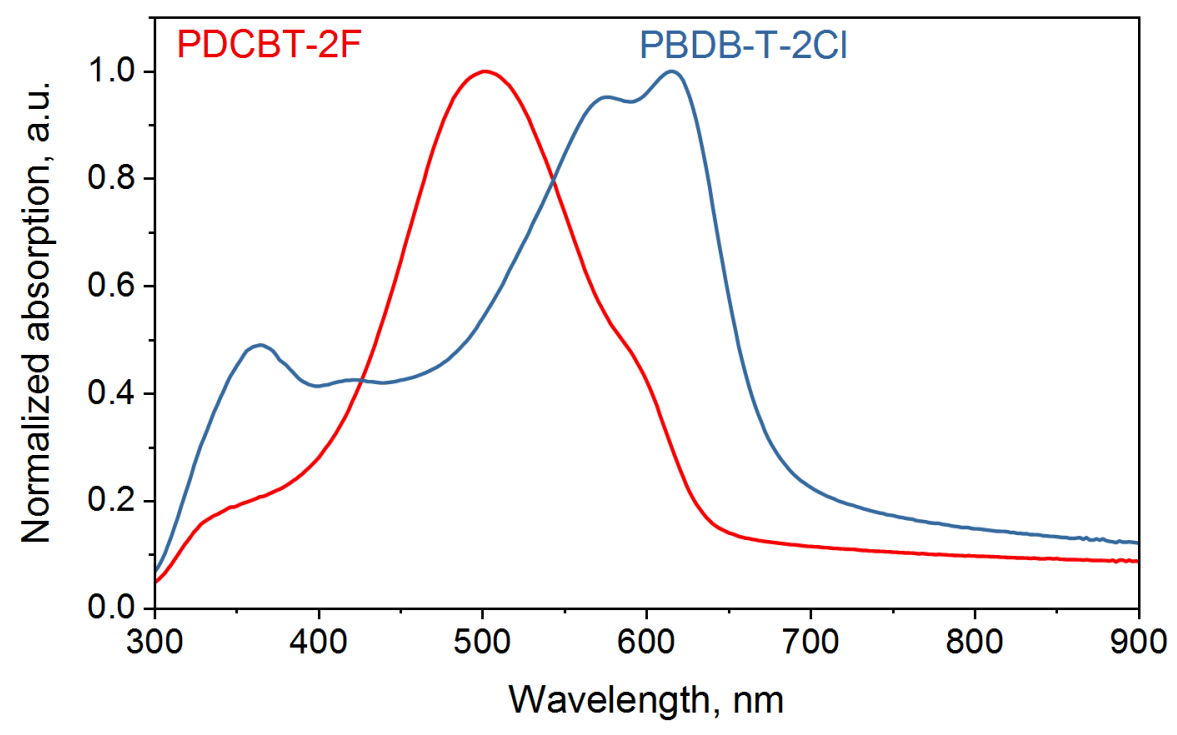

Fig. S1 PBDB-T-2Cl and PDCBT-2F absorption spectra.

a)

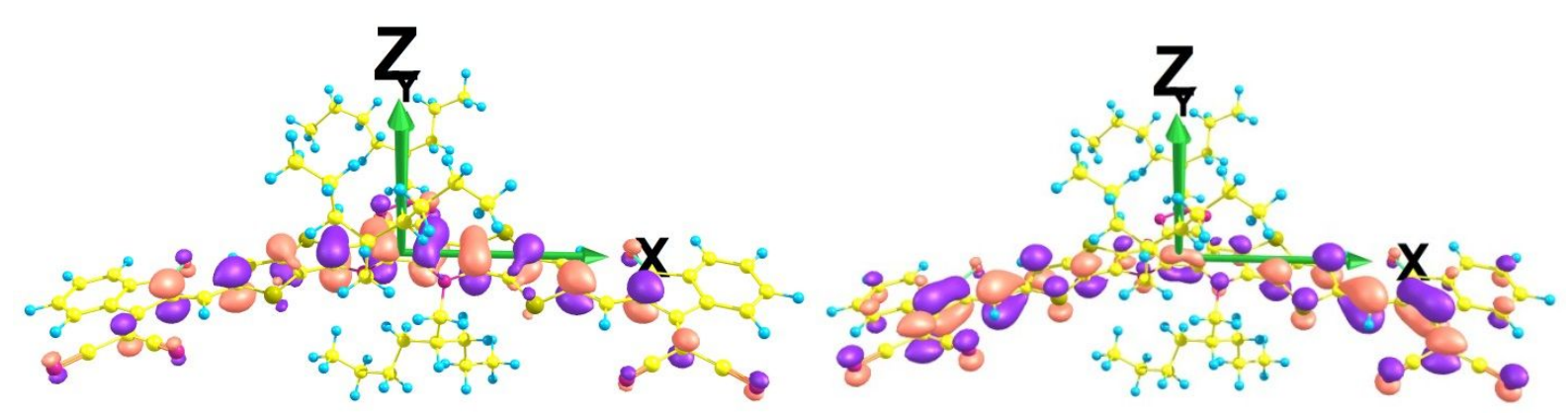

Fig. S2 a) HOMO and b) LUMO orbitals of $Y 1$ in SO state. 
a)

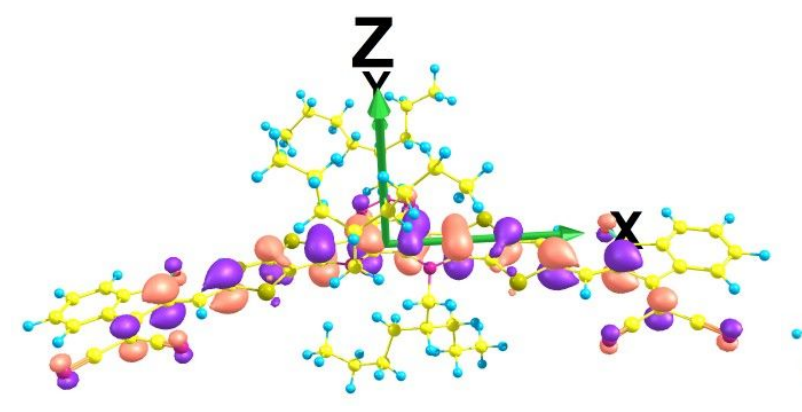

c) b)



d)

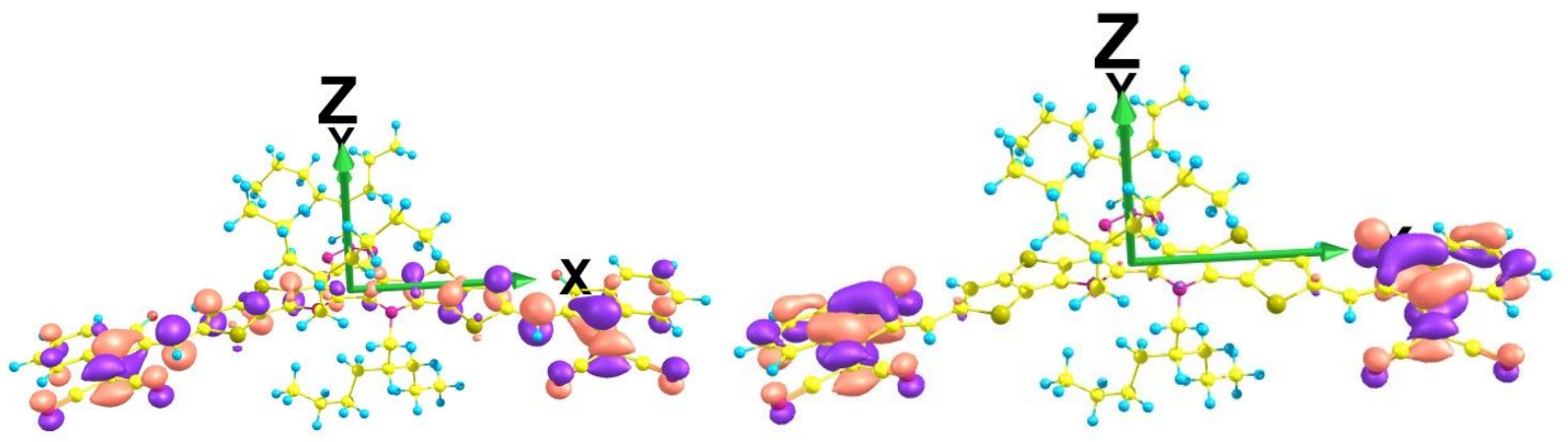

Fig. S3 a) HOMO; b) SOMO; c) LUMO and d) LUMO+1 orbitals of Y1 -1 ion in the ground state.

During computations density functional theory (DFT) and time dependent density functional theory (TDDFT) methods were used. Computations were performed at the b3lyp/6-31G(d,p) level using G09 [ ] code. Solvent effects for chloroform solution was accounted using polarizable continuum model as it is implemented in G09 code.

Geometry of neutral Y1 molecule (charge $=0$ ) was optimized for ground electronic state in vacuum S0 only. The optimized neutral Y1 structure in S0 state is shown in (Fig S4).

Geometry optimization for Y1 -1 ion (doublet D1 state) was not performed and all computations of D1 were performed using $\mathrm{Y} 1 \mathrm{~S} 0$ ground state geometry. 

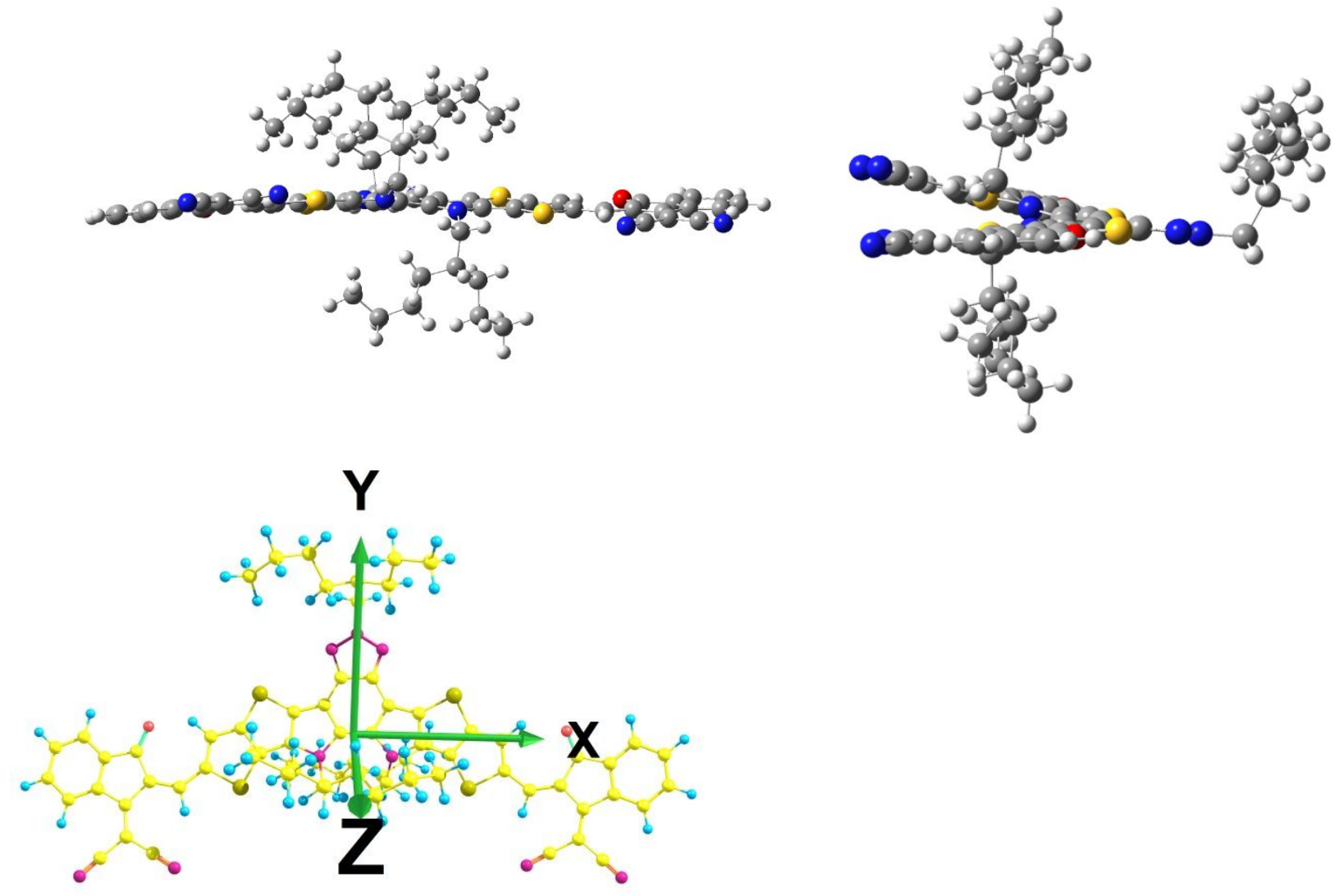

Fig. S4 SO optimized structure of neutral Y1 molecule.

Table S1. Y1 molecule SO state and Y1 -1 ion (doublet D1 state) electronic excitation characteristics (for most active excitations). All computations were performed using $Y 1$ molecule in vacuum optimized geometry for SO state, solvent effects accounted where it is stated.

\begin{tabular}{|l|c|c|c|}
\hline \multicolumn{1}{|c|}{ Molecule } & $\begin{array}{c}\text { S0-> S1 excitation } \\
\text { energy, } \mathbf{~ e V ~}(\mathbf{n m})\end{array}$ & $\begin{array}{c}\text { Oscillator } \\
\text { strength }\end{array}$ & $\begin{array}{c}\text { Excitation } \\
\text { character }\end{array}$ \\
\hline & & & \\
\hline Y1, S0 state & $1.818(682.2)$ & 2.16 & HOMO->LUMO \\
\hline $\begin{array}{l}\text { Y1, S0 state } \\
\text { (in chloroform solution, S0 in vacuum } \\
\text { geometry) }\end{array}$ & $1.668(743.1)$ & 2.45 & HOMO->LUMO \\
\hline & & & \\
\hline Y1, D1 state & $0.5565(2227.8)$ & 0.668 & SOMO->LUMO \\
\hline Y1, D1 state & $1.696(730.9)$ & 1.08 & HOMO->SOMO \\
\hline Y1, D1 state & $2.174(570.4)$ & 0.132 & HOMO->LUMO \\
\hline & & & \\
\hline Y1, D1 state (in chloroform solution, S0 & $0.4171(2972.6)$ & 0.897 & SOMO->LUMO \\
\hline
\end{tabular}




\begin{tabular}{|l|c|c|c|}
\hline in vacuum geometry) & & & \\
\hline $\begin{array}{l}\text { Y1, D1 state (in chloroform solution, S0 } \\
\text { in vacuum geometry) }\end{array}$ & 1.6318 (759.8) & 1.20 & HOMO->SOMO \\
\hline $\begin{array}{l}\text { Y1, D1 state (in chloroform solution, S0 } \\
\text { in vacuum geometry) }\end{array}$ & 2.1083 (588.1) & 0.256 & HOMO->LUMO \\
\hline
\end{tabular}

The Y1 SO->S1 excitation has HOMO LUMO character. HOMO and LUMO orbitals (fig. S2) show that during this excitation electronic charge is transferred from the central molecular part to the ends of the PI system along the $\mathrm{X}$-axis (Fig. S4).

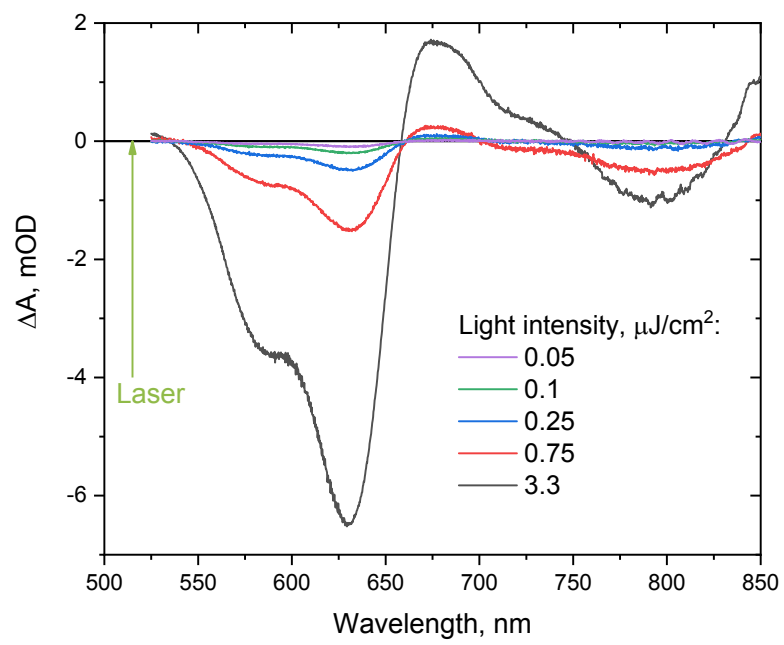

Fig. S5 Transient absorption spectra at 1ps delay time of PBDB-T:Y1 sample at various $515 \mathrm{~nm}$ laser excitation intensites. 


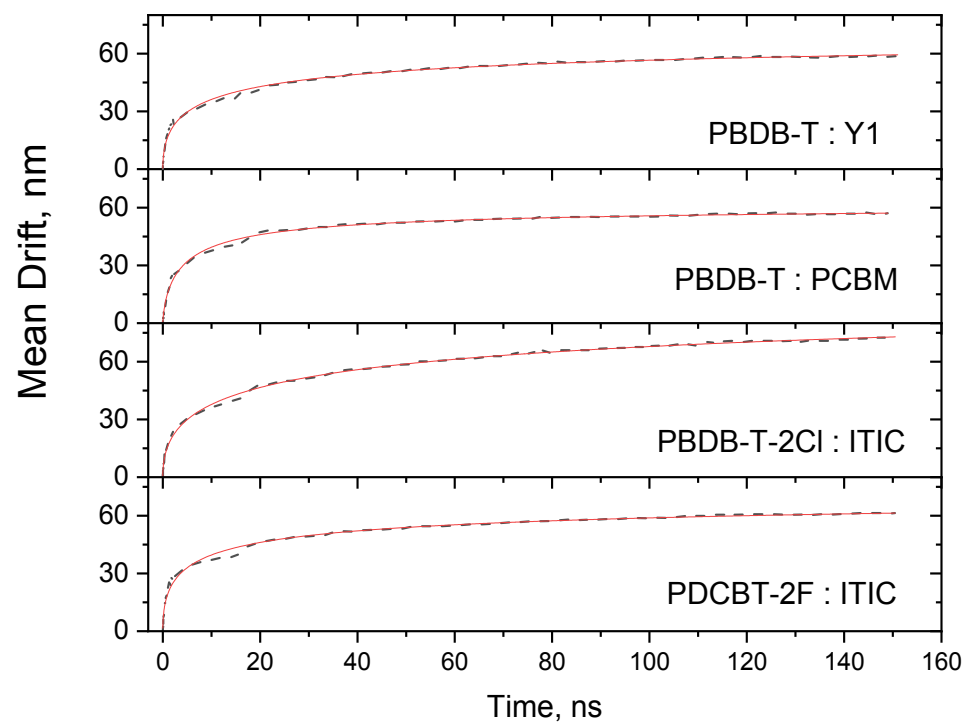

Fig. S6 Mean carrier drift kinetics (black dotted curves) obtained from transient photocurrent $\left(U_{G E N}=-2 \mathrm{~V}\right)$ measurements. Fitted curves (red solid lines) were used to evaluate mobility kinetics in 2-50ns time window.

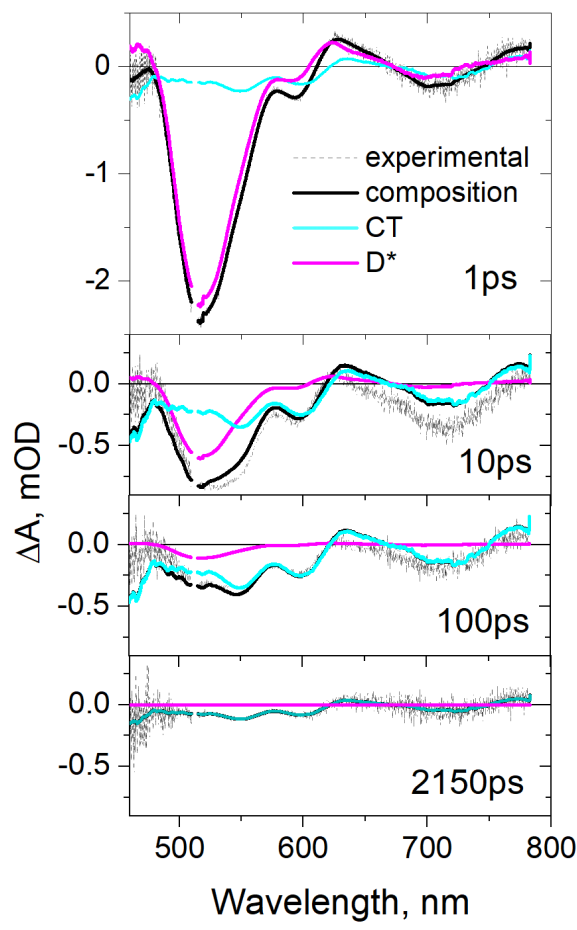

Fig. S7 Decomposition of the TA spectra of the PDCBT-2F:ITIC blent into donor excited state( $\left.D^{*}\right)$ and $C T$ state components. 


\section{REFERENCES:}

[1] Devižis, A.; De Jonghe-Risse, J.; Hany, R.; Nüesch, F.; Jenatsch, S.; Gulbinas, V.; Moser, J.-E. Dissociation of Charge Transfer States and Carrier Separation in Bilayer Organic Solar Cells: A TimeResolved Electroabsorption Spectroscopy Study. J. Am. Chem. Soc. 2015, 137 (25), 8192-8198. https://doi.org/10.1021/jacs.5b03682.

[2] Devižis, A.; Hertel, D.; Meerholz, K.; Gulbinas, V.; Moser, J.-E. Time-Independent, High Electron Mobility in Thin PC 61 BM Films: Relevance to Organic Photovoltaics. Organic Electronics 2014, 15 (12), 3729-3734. https://doi.org/10.1016/j.orgel.2014.10.028. 